\title{
Efficacy of enalapril in essential hypertension and its comparison with atenolol
}

\author{
M. El Mangoush, N.K. Singh, S. Kumar, A. Basha, B.S. Gupta ${ }^{1}, Y . K$. Bolya \\ and A. Gamati
}

Departments of Medicine and ${ }^{1}$ Community Medicine, Hawari Hospital, Arab Medical University, Benghazi, Libya.

\begin{abstract}
Summary: The effect of enalapril was evaluated in 67 patients with essential hypertension, and its therapeutic efficacy was compared with atenolol in a placebo run-in, single-blind, cross-over trial. Enalapril significantly reduced blood pressure in all grades of essential hypertension. As monotherapy it 'normalized' blood pressure in $88 \%, 50 \%$ and $25 \%$ of patients with mild, moderate and severe hypertension respectively. Optimal dose for most of the patients was 20 to $\mathbf{4 0} \mathrm{mg} /$ day. Comparison with atenolol revealed almost parallel efficacy of the two drugs, although enalapril produced a significantly greater reduction in systolic blood pressure in patients with mild and moderate hypertension $(\mathbf{P}<0.01$ in each group). No serious side effects were encountered with either drug. Enalapril, therefore, has a potent and slightly superior antihypertensive effect to that of atenolol, and may be used as a 'first-step' drug in the treatment of hypertensive patients.
\end{abstract}

\section{Introduction}

Enalapril is a relatively new, long-acting, nonsulphydryl angiotensin converting enzyme (ACE) inhibitor with a few side effects. ${ }^{1,2}$ In placebocontrolled trials, it has been reported to be effective in controlling blood pressure (BP) in about 50 to $75 \%$ patients with mild to moderate hypertension. ${ }^{3-5}$ However, most of the clinical trials have been done with a fixed dose of $20 \mathrm{mg}$ or $40 \mathrm{mg}$ per day. Blood pressure response with increasing doses of enalapril has been correlated by only a few workers, ${ }^{6,7}$ and its optimal dose has not been elucidated. Moreover, only a few studies have been done to evaluate its therapeutic efficacy as a monotherapeutic agent in patients with severe hypertension. ${ }^{8}$

Comparative studies on the antihypertensive effect of enalapril with beta blockers propranolol, ${ }^{9,10}$ metoprolol $^{11}$ and atenolol ${ }^{8,12}$ have shown these drugs to be of almost similar efficacy. However, the place of enalapril as a 'first-step' antihypertensive drug has not yet been clearly established. We selected atenolol for comparison as it is a conventional first-line antihypertensive drug and because of the limited reports available on the comparative efficacy of the two drugs. ${ }^{8,12}$

Correspondence: M. El Mangoush, M.D., F.R.C.P., P.O. Box 2583, Benghazi, Libya

Accepted: 21 September 1989
The objective of the present study was two fold: (i) to evaluate the antihypertensive efficacy and tolerance of increasing doses of enalapril in patients with essential hypertension of severity ranging from mild to severe, and to find out its optimal therapeutic dose; and (ii) to compare the efficacy and safety of enalapril with that of atenolol in order to evaluate the place of enalapril as a 'first-step' drug in hypertensive patients.

\section{Materials and methods}

\section{The patients}

Sixty seven patients with essential hypertension completed the trial. All patients gave informed consent and were randomly selected from the hypertension clinic. Patients with secondary or malignant hypertension, pregnancy, heart failure, heart block, diabetes mellitus, bronchial asthma or peripheral vascular disease and significant renal or hepatic dysfunction were not included in the study. All patients underwent a complete physical examination, chest X-ray, electrocardiogram, blood biochemistry and urine analysis. Depending on diastolic blood pressure (DBP), patients were classified into mild $(90-104 \mathrm{mmHg})-25$ cases, moderate $(105-120 \mathrm{mmHg})-30$ cases, and severe hypertension $(>120 \mathrm{mmHg})-12$ cases. There 
were 33 males and 34 females, aged 28 to 65 years $(48.30 \pm 2.34)$. Average duration of hypertension was $5.7 \pm 1.1$ years with a range of 1 month to 18 years.

\section{Study design}

This was a placebo run-in, single-blind, cross-over study for 8 to 10 weeks with each of enalapril and atenolol. After withdrawal of their previous antihypertensive treatment, all patients received placebo ( 1 tablet daily) for 4 weeks as a 'washout' period. Patients were then randomly assigned to once daily treatment with either enalapril or atenolol. Starting dose of enalapril was $10 \mathrm{mg} /$ day for mild and $20 \mathrm{mg} /$ day for moderate and severe hypertension. Depending on therapeutic response, it was increased at 2-weekly intervals to 20 to 40 to 60 to $80 \mathrm{mg} /$ day unless diastolic BP was reduced below $90 \mathrm{mmHg}$ or the patient developed side effects of the drug. Starting dose of atenolol was $50 \mathrm{mg} /$ day for mild hypertension which was increased to $100 \mathrm{mg} /$ day after 4 weeks if required. Patients with moderate and severe hypertension received a fixed dose of $100 \mathrm{mg} /$ day for 8 weeks or less if diastolic BP was 'normalized'. While crossing-over to the other drug, all patients received an intervening placebo for 4 weeks as washout period. The trial was considered to be complete if diastolic BP was normalized $(<90 \mathrm{mmHg})$ or after the patient had received the maximum dose of enalapril $(80 \mathrm{mg} /$ day $)$ and atenolol $(100 \mathrm{mg} /$ day $)$, each for at least 4 weeks.

All patients attended the clinic at 2-weekly intervals, and earlier if required. At each visit blood pressure was measured and side effects objectively enquired. All blood pressure measurements were made uniformly after rest for at least 10 minutes, in the sitting position, in the right arm, using a random zero mercury sphygmomanometer almost at the same time (between 16.00 and $18.00 \mathrm{~h}$ ) on each visit. The first and fifth Korotkoff sounds were used to determine systolic and diastolic BP respectively. 'Response' to therapy refers to a reduction in diastolic BP of $>10 \mathrm{mmHg}$ or to $<90 \mathrm{mmHg}$, and 'normotension' or 'normalization' of BP refers to diastolic $\mathrm{BP}<90 \mathrm{mmHg}$.

\section{Statistical analysis}

Mean BP was calculated as diastolic BP plus one third of systolic minus diastolic BP. All data are shown as mean \pm standard error of mean (s.e.m.) values. Statistical analysis to compare the antihypertensive effect of enalapril and atenolol included ' $t$ ' test applied to paired comparison. ${ }^{13} \mathrm{~A}$ $P$ value of $<0.05$ was considered as significant and $P$ value of $<0.01$ as highly significant.
The power of the test for the mild, moderate and severe groups was SBP 0.5-0.6, 0.6-0.7 and $0.3-0.4$, DBP $0.1-0,<0.1,0.3-0.4$ and $0.1-0.2$, and MBP $0.8-0.9,0.3-0.4,0.3-0.4$ respectively.

\section{Results}

\section{Relation to severity of hypertension}

Response to enalapril treatment was noticed in all patients with mild, $20(66.6 \%)$ with moderate and $7(58.3 \%)$ patients with severe hypertension; whereas 'normalization' of BP was achieved in $22(88 \%)$ with mild, $15(50 \%)$ with moderate and $3(25 \%)$ patients with severe hypertension. The dose of enalapril required to 'normalize' the BP was $10 \mathrm{mg}$ in $5,20 \mathrm{mg}$ in $22,40 \mathrm{mg}$ in 17 and $60 \mathrm{mg}$ in 6 patients. Increasing the dose further to $80 \mathrm{mg} /$ day, however, did not produce any additional beneficial effect.

Response to atenolol treatment occurred in all patients with mild, $26(86.6 \%)$ with moderate and $7(58.3 \%)$ patients with severe hypertension, blood pressure was 'normalized' in $23(92 \%), 13(43.3 \%)$ and $2(16.6 \%)$ patients respectively. The dose of atenolol required to 'normalize' BP was $50 \mathrm{mg}$ in 12 and $100 \mathrm{mg}$ in 26 patients.

\section{Effect on entire patient group}

There was a significant reduction in mean \pm s.e.m. values of systolic, diastolic and mean BP in all grades of hypertension with both drugs as compared to placebo (Table I). The magnitude of reduction in BP was slightly greater with enalapril (13 to $16 \%$ ) as compared to atenolol ( 7 to $15 \%$ ). Comparison of their antihypertensive effect revealed no significant difference in diastolic BP but reduction in systolic BP was significantly greater with enalapril than atenolol in both mild and moderate hypertension groups $(P<0.01$ in each group) as shown in Figures 1 and 2. In patients with severe hypertension, the fall in BP was relatively more with enalapril than atenolol but the difference was statistically insignificant.

The pulse rate for the entire group of 67 patients before and after enalapril treatment was $75.5 \pm 1.6$ and $76.4 \pm 1.7(P=$ not significant $)$, whereas with atenolol treatment it decreased from $76.2 \pm 1.8$ to $66.8 \pm 1.5(P<0.001)$.

\section{Side effects}

On enalapril treatment 2 patients each had headache and somnolence and 1 had dizziness whereas with atenolol there was dizziness in 2 and heart failure in 1 patient. 

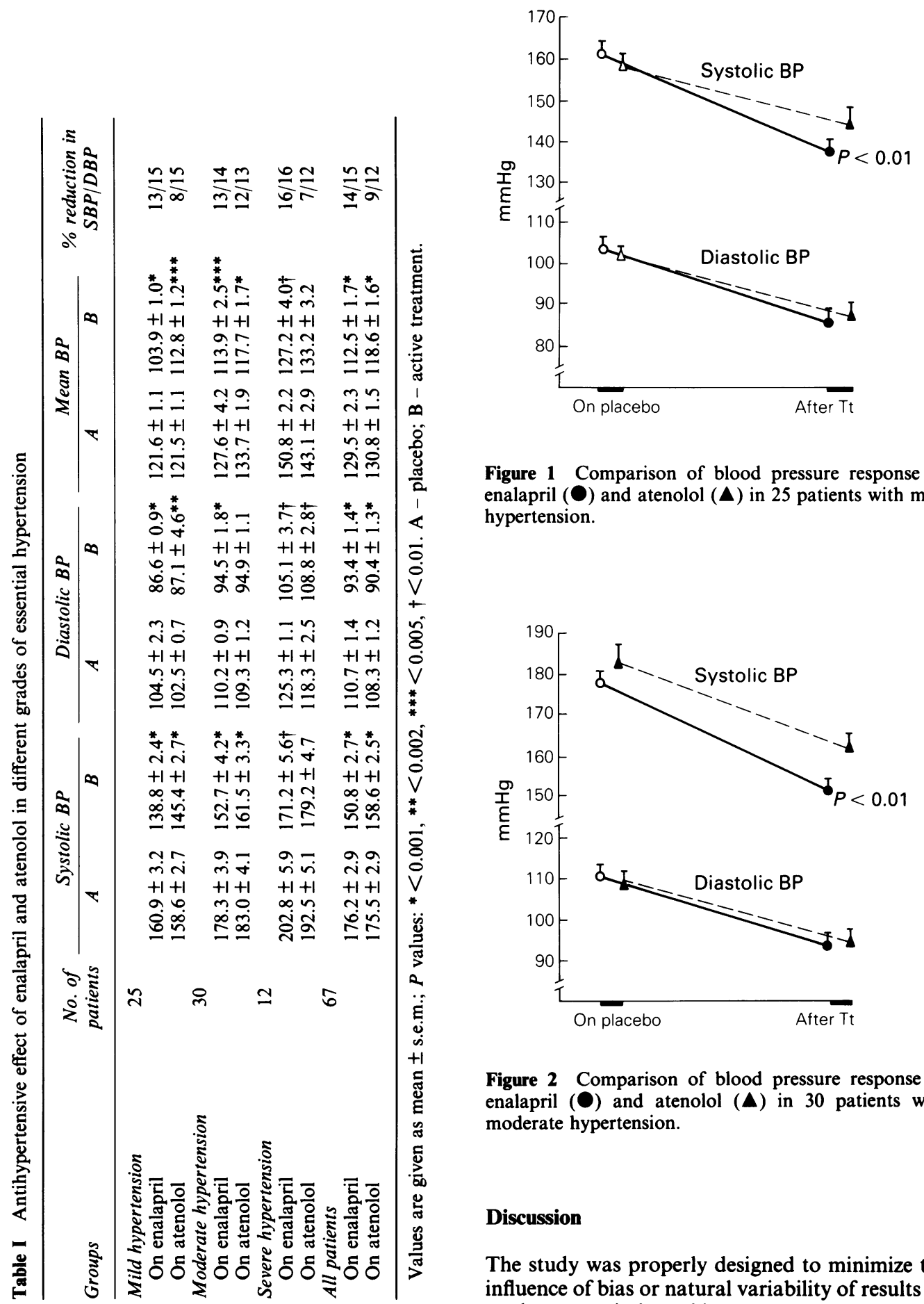

Figure 1 Comparison of blood pressure response to enalapril (O) and atenolol $(\Delta)$ in 25 patients with mild hypertension.

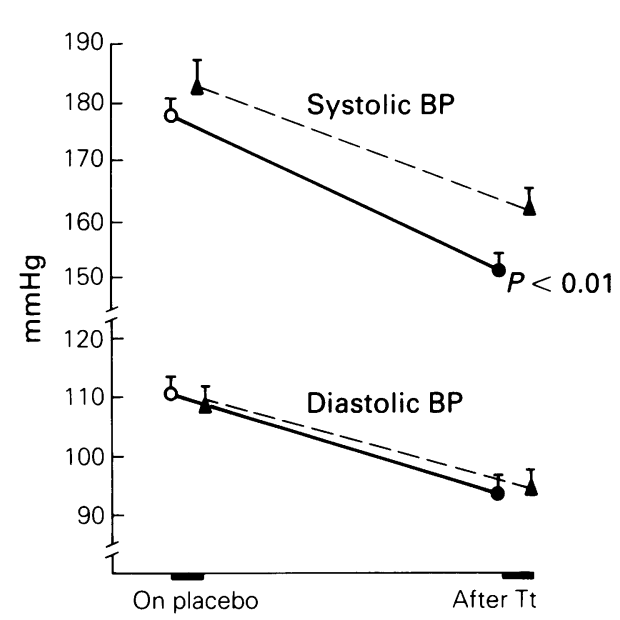

Figure 2 Comparison of blood pressure response to enalapril $(O)$ and atenolol $(\Delta)$ in 30 patients with moderate hypertension.

\section{Discussion}

The study was properly designed to minimize the influence of bias or natural variability of results by wash-out periods, taking BP measurements in uniform situations using a random zero mercury sphygmomanometer and adopting clearly defined criteria. A placebo run-in washout period of more than 4 weeks was considered undesirable because 
of the attendant risks of uncontrolled hypertension.

Our results of enalapril therapy in patients with mild to moderate hypertension are comparable to those reported earlier. ${ }^{3-5}$ Enalapril alone failed to control BP in a majority of the patients with severe hypertension. The optimal dose of enalapril for most of the patients, who achieved normalization of BP, was $20 \mathrm{mg}$ to $40 \mathrm{mg} /$ day. A smaller dose $(10 \mathrm{mg} /$ day) was effective in only a few patients with mild hypertension, and increasing the dose to $60 \mathrm{mg} /$ day benefited only a few patients. Only a few previous studies ${ }^{6,7}$ have compared the BP response with different doses of enalapril administered.

Comparative studies of enalapril with beta blockers in patients with mild to moderate hypertension have revealed it to be as effective as propranolol, ${ }^{9,10}$ metoprolol $^{11}$ and atenolol. ${ }^{8,12}$ In the present study, comparison of enalapril and atenolol revealed almost parallel efficacy of the 2 drugs in all grades of hypertension, although the per cent reduction in BP with enalapril was superior to atenolol. Moreover, reduction in systolic BP was more marked with enalapril than atenolol in patients with both mild and moderate hypertension $(P<0.01$ in each group). Similar findings have been reported by other workers. ${ }^{8,12}$ The greater reduction in systolic BP with enalapril compared with atenolol appears clinically important as there is now greater evidence to suggest that

\section{References}

1. McFate, S.W., Kulaga, S.F., Moncloa, F., Pingaoni, R. \& Walker, J.F. Overall tolerance and safety of enalapril. $J$ Hypertens 1984, 2(Suppl 2): 113-117.

2. Moncloa, F., Sromovsky, J.A., Walker, J.F. \& Davies, R.D. Enalapril in hypertension and congestive heart failure. Overall review of efficacy and safety. Drugs 1985, 30(Suppl 1): 82-89.

3. Lund-Johensen, P. \& Omvik, P. Long-term haemodynamic effects of enalapril (alone and in combination with hydrochlorthiazide) at rest and during exercise in essential hypertension. J Hypertens 1984, 2(Suppl 2): 49-56.

4. Sassano, P., Chatellier, G., Alhenc-Gelas, F., Carvol, P. \& Menard, J. Antihypertensive effect of enalapril as first-step treatment of mild and moderate uncomplicated essential hypertension. Am J Med 1984, 7: 18-22.

5. Fouad, F.M., Tarazi, R.C., Bravo, E.L. \& Textor, S.C. Haemodynamic and antihypertensive effects of the new oral angiotensin-converting-enzyme inhibitor MK-421 (enalapril). Hypertension 1984, 6: 167-174.

6. Davies, R.O., Irvin, J.D., Kramsch, D.K., Walker, J.P. \& Moncloa, F. Enalapril worldwide experiences. Am J Med 1984, 77: 23-35.

7. Bergstand, R., Herlitz, H., Johansson, S. et al. Effective dose range of enalapril in mild to moderate essential hypertension. Br J Clin Pharmacol 1985, 19: 605-611.

8. Arr, S.M., Burgees, J., Cooper, W.D. et al. A comparative study of enalapril and atenolol in moderate to severe hypertension. Br J Clin Pharmacol 1984, 18: 290-292. increased systolic rather than diastolic BP is predictor of cardiovascular morbidity. ${ }^{14,15}$ Moreover, it may prove to be a useful drug for the treatment of isolated systolic hypertension. Further study is required in this direction.

Side effects of enalapril were mild and trivial in the present study, and were unrelated to its dose. Its reported side effects were 0.5 to $5 \%$ and included headache, dizziness, fatigue, diarrhoea, skin rash, nausea, taste disturbance and, rarely, symptomatic hypotension. . $^{1,26}$

In conclusion, enalapril was found to possess potent antihypertensive effect. It controlled BP in the majority of patients with mild to moderate hypertension at a dose of 20 to $40 \mathrm{mg} / \mathrm{day}$. It was well tolerated, and has a parallel or slightly superior antihypertensive effect as compared to atenolol. Moreover, it has no limitations to its use with beta blockers as observed in conditions such as heart failure, heart block, bronchial asthma, diabetes mellitus and peripheral vascular disease. Enalapril is, therefore, clearly an effective and safe alternative first-line drug in the 'stepped-care' approach to the treatment of hypertension.

\section{Acknowledgements}

Merck Sharp \& Dohme (MSD) kindly supplied enalapril. We thank Miss Z.D. Vesna for typing the paper.
9. Simon, A.C., Levenson, J.A., Bouthier, J., Manrek, B. \& Safar, M.E. Comparison of oral MK-421 and propranolol and their effects on arterial and venous vessels of the forearm. Am J Cardiol 1984, 53: 781-785.

10. Enalapril in Hypertension Study Group (UK). Enalapril in essential hypertension: A comparative study with propranolol. Br J Clin Pharmacol 1984, 18: 51-56.

11. O'Connor, D.T., Mosley, C.A., Cervenka, J. \& Bernstein, K.N. Contrasting haemodynamic responses to the angiotensin enzyme inhibitor enalapril and the beta-andrenergic antagonist metoprolol in essential hypertension. $J$ Hypertens 1984, 2(Suppl 2): 89-92.

12. Helgeland, A., Strommen, R., Hagelund, C.H. \& Tretli, S. Enalapril, atenolol and hydrochlorthiazide in mild to moderate essential hypertension. A comparative multicentre study in general practice in Norway. Lancet 1986, i: 872-875.

13. Bradford Hill, A. Principles of Medical Statistics, 9th edition. The Lancet Limited, London, 1971, pp. 141-151.

14. Kannel, W.B., Dawber, T.R. \& McGee, D.L. Prospectives of systolic hypertension. Circulation 1981, 61: 1179-1182.

15. Lichtenstein, M.J., Shipley, M.J. \& Rose, G. Systolic and diastolic blood pressure as predictors of coronary heart disease mortality in Whitehall study. $\mathrm{Br} M e d \mathrm{~J}$ 1985, 29: 243-245. 